\title{
A comparison of approximate techniques for the determination of potential energy surfaces of lon-molecule charge transfer systems
}

\author{
B. Ramiro Diaz a) and P. Wahnon \\ Depto. TEAT, E.T.S.I. Telecommunicacion, U.P.M. Ciudad Universitaria s/n, 28040 Madrid, Spain \\ V. Sidis \\ Laboratoire des Collisions Atomiques et Moléculaires, Université Paris XI, URA 281, Bât. 351, \\ 91405 Orsay, France
}

\begin{abstract}
The $\left(\mathrm{HO}_{2}\right)^{+}$molecular ion is used to experiment two approximate procedures which aim at reducing the computation effort that is needed for the determination of potential energy surfaces of ion-molecule charge transfer systems. The two procedures involve configuration interaction $(\mathrm{CI})$ calculations of moderate sizes and are based on diagonal corrections of the electronic Hamiltonian matrix in a basis of projected-valence bond (PVB) configuration-state functions (CSF). The PVB-CSF's used in practice correspond to a full valence CI for each ionic or neutral partner as well as single excitations accounting for polarization and electron transfer. The diagonal corrections are of two sorts: (i) if insufficiently large orbital expansion bases are used they remove the relative ion-molecule basis set superposition error; (ii) if asymptotic energy levels of the involved neutrals or ions in their ground or valence excited states are misplaced they properly adjust these levels. When applied to $\left(\mathrm{HO}_{2}\right)^{+}$using a minimal or an extended orbital basis set expansion the proposed approaches yield concording results. The results also agree with the effective model potential (EMP) data of Grimbert et al. [Chem. Phys. 124, 187 (1988)] which have proved successful in the description of the $\mathrm{H}^{+}$ $+\mathrm{O}_{2}$ charge transfer dynamics. Comparison with fragmentary results from MRD-CI calculations by Vazquez et al. [Mol. Phys. 59, 291 (1986)] and Schneider et al. [Chem. Phys. 128, 311 (1988)] is somewhat mitigated. The method should be particularly useful for bulky ionmolecule systems.
\end{abstract}

\section{INTRODUCTION}

The phenomenon of charge migration from one part of a molecular system to another is a problem of great current interest. ${ }^{1}$ The detailed investigation of state-to-state ionmolecule charge transfer reactions ${ }^{2}$ may provide basic information on the dynamics of this phenomenon. Recent work has shown that the theory of nonadiabatic molecular collisions is capable of successfully treating those charge transfer problems provided appropriate quantum chemical data may be accessed easily. ${ }^{2}$ This point is crucial to catch the philosophy of the present work. Basically, the required information consists of electronic structural properties of the supersystem made of all the reaction partners for fixed nuclear geometries; those are mainly potential energy surfaces and relevant couplings. ${ }^{2,3}$ These properties are needed for, at least, two electronic states of the system, namely, the one correlating with the reactants and that correlating with the products. Sometimes, additional states that mediate the reaction may also be necessary. It is well known that distant charge transfer reactions at low energy are highly sensitive to the energy separation of the interacting states. Hence, the least specification placed on those states is to correctly give the so-called resonance energy defect, defined as the difference between the ionization potential of

\footnotetext{
- Permanent address: Dpto. Aerotecnica, E.U.I.T. Aeronauticos, Ciudad Universitaria s/n, 28040 Madrid, Spain.
}

the neutral and the recombination energy of the ion. This requirement is not as trivial as it may appear at first sight since it implies to have as accurate a description of the energy surface of a neutral reagent molecule as that (those) of its ion(s); state-of-the-art calculations come up against that difficulty (see e.g., Ref. 4, Sec. II and Sec. IV B 2). Another specification has to do with polarizationtype distortions. Many molecules own a number of valence states lying, within a few $\mathrm{eV}$, in the neighborhood of the ground state. When placed in the field of the incoming ion those states admix to the ground state thereby lowering its energy level. The correct relative placing of the energy surfaces of low lying valence states of the neutral partner in thus seen to be a necessary provision of the calculations that are to produce the sought quantum chemical data. Ideally these data would be prepared in a diabatic representation ${ }^{3}$ in order to facilitate their use in the quantitative description of the dynamics and last, but not least, they should be made available for a dense grid of the relative nuclear coordinates (in a nondissociative $\mathrm{A}^{+}+\mathrm{BC}$ collision system with $\mathrm{B} \equiv \mathrm{C}$ the number of grid points is in the range of a few thousands and an incompressible minimum is in the $10^{2}$ range). Despite of a steady evolution over the past decade of both computational strategies and computer efficiency, the systematic determination of quantum chemical data bearing all the above specifics by state-of-the-art $a b$ initio techniques is presently out of reach. The latter techniques furnish benchmark, albeit fragmentary, information, while less orthodox means palliate the rest. The present work belongs to the latter category of approaches. 
Our initial objective when we started this work was to investigate $\left(\mathrm{RG}-\mathrm{O}_{2}\right)^{+}$charge transfer systems, where RG is a heavy rare gas $(\mathrm{Ar}, \mathrm{Kr})$. These systems have been the subjects of recent experimental studies. ${ }^{5}$ So far, no realistic method exists to supply the data needed to investigate the charge transfer dynamics of those systems. Of the two manageable methods that were recently tried on the $\left(\mathrm{H}-\mathrm{O}_{2}\right)^{+}$system, the diatomics-in-molecules (DIM) method $^{6}$ was found to meet with problems, ${ }^{7}$ while the effective model potential (EMP) ${ }^{8}$ approach has not yet been extended beyond ( $\mathrm{H}$-molecule $)^{+}$systems. It was thus decided to look for approaches that make quantum chemistry technology manageable at the expense of some compromise with the strict observance of "ab initio' ism." This decision was arrived at after it was realized that in systems like $\left(\text { atom }-\mathrm{O}_{2}\right)^{+}$a large part of the computation effort was being spent in trying to describe the $\mathrm{O}_{2} / \mathrm{O}_{2}^{+}$groups in the triatomic complex (see Sec. II B). Since there is ample experimental and theoretical information on those groups the strategy consisted in treating them in an ad hoc manner. In this paper we experiment two approaches on the $\left(\mathrm{H}-\mathrm{O}_{2}\right)^{+}$system which has been chosen, on account of the availability of various pieces of information..$^{6-11}$ Moreover, this system illustrates particularly well the sorts of problems we endeavored to address. Extension of the present proposals to the more complex $\left(\mathrm{RG}-\mathrm{O}_{2}\right)^{+}$systems is underway.

\section{FEATURES OF THE PRESENT APPROACH}

\section{A. The PVB description}

The first characteristics of our approaches is that they make use of a valence-bond (VB)-type description of the atom-molecule system. As discussed elsewhere ${ }^{3}$ such a description was preferred because it is well suited to carry through certain techniques designed to generate diabatic states by emphasizing characters of the isolated reaction partners. This feature makes a VB-type description particularly well suited to controlling the work to be done on the specific $\mathrm{O}_{2} / \mathrm{O}_{2}^{+}$groups in a general (atom- $\left.\mathrm{O}_{2}\right)^{+}$triatomics.

Throughout, use will thus be made of so-called projected valence bond (PVB) $)^{3,8,12}$ configuration state functions (CSF) built from self-consistent field (SCF) orbitals of the isolated $\mathrm{O}_{2}$ or $\mathrm{H}$ neutral species; those orbitals are orthogonalized $^{3,8,12}$ by the Schmidt procedure in the following order:

$1 \sigma_{g}, 1 \sigma_{u}, 2 \sigma_{g}, 2 \sigma_{u}, 3 \sigma_{g}, 1 \pi_{u}, 1 s, 1 \pi_{g}, \forall \mathrm{VO}_{\mathrm{O} 2}, \forall \mathrm{VO}_{\mathrm{H}}$.

Diatomic molecular orbital (MO) labels refer to $\mathrm{O}_{2}$, while atomic orbital (AO) labels refer to $\mathrm{H}$; VO refers to the selected virtual orbitals of $\mathrm{O}_{2}$ or $\mathrm{H}$. The computational procedure is actually a PVB-CI one. In this procedure the CI (configuration interaction) aims at describing: (i) the interaction between $\mathrm{H}^{+}$(or $\mathrm{H}$ ) and the $\mathrm{O}_{2}$ (respectively, $\mathrm{O}_{2}^{+}$) group, and, (ii) the $\mathrm{O}-\mathrm{O}$ interaction within that group. Considering the latter aspect, when the $\mathrm{H}$ center is located at a large distance $R$ from the molecule, $\mathrm{CI}$ is required to describe, among other things, the correct dis-
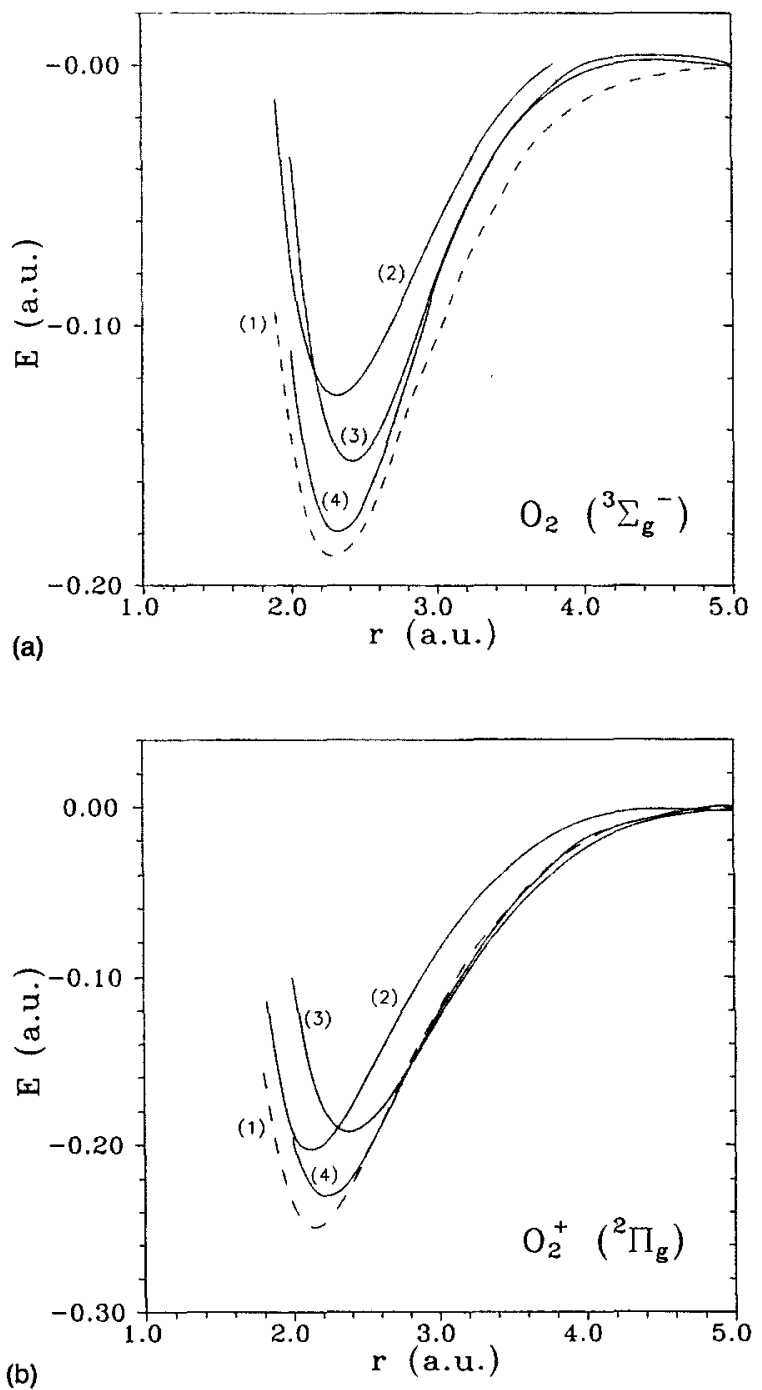

FIG. 1. Ground state potential energy curve for (a) $\mathrm{O}_{2}$ and (b) $\mathrm{O}_{2}^{+}$. The labeling of the curves is as follows: (1) data from Ref. 21 ; (2) SCF-CI result of Sec. II B using the [11,6/5,4] basis of Ref. 13; (3) SCF-CI result of Sec. II B using the STO-6G expansion of the single- $\xi$ basis of Ref. 15; (4) same as curve (3) with the inclusion of a $d$ polarization orbital $\left(\zeta_{d}=0.8\right)$.

sociation of the $\mathrm{O}_{2} / \mathrm{O}_{2}^{+}$states into proper atomic states at large $\mathrm{O}-\mathrm{O}$ distances. Moreover, it is intended to enable one to describe at once $\mathrm{O}_{2}^{(*)}$ and $\mathrm{O}_{2}^{+(*)}$ states with a unique set of SCF-MO.

\section{B. Difficulties with the $\mathrm{O}_{2} / \mathrm{O}_{2}^{+}$groups}

It is often recommended that molecular orbitals be expanded in terms of at least double- $\zeta+$ polarization basis sets centered on each atom. However, such an expansion makes it very difficult to accurately describe the equilibrium properties of the $\mathrm{O}_{2} / \mathrm{O}_{2}^{+}$diatomics. This is illustrated in Figs. 1(a) and 1(b) and Table I (col. 2) by calculations on $\mathrm{O}_{2}$, resp. $\mathrm{O}_{2}^{+}$, involving

(i) for the SCF step: Dunning's [11,6/5,4] CGTO basis $^{13}$ plus a $d$ polarization orbital $\left(\zeta_{d}=1.26\right)$; 
TABLE I. Comparison of some $\mathrm{O}, \mathrm{O}_{2}$, and $\mathrm{O}_{2}^{+}$properties.

\begin{tabular}{|c|c|c|c|c|c|}
\hline & Experiment ${ }^{2}$ & $\begin{array}{c}{[11,6 / 5,4]+d^{b}} \\
(\ddagger)\end{array}$ & $\begin{array}{l}\text { MRD-CI } \\
(\mathrm{FCI})\end{array}$ & $\begin{array}{l}\text { STO-6G } \\
(+d)\end{array}$ & STO- $6 \mathrm{G}_{\mathrm{sc}}+d^{t}$ \\
\hline$D_{e}(\mathrm{eV})$ & 5.21 & 3.45 & $\begin{array}{c}4.536 \\
(4.903)\end{array}$ & $\begin{array}{c}4.14 \\
(5.00)\end{array}$ & 4.88 \\
\hline$r_{e}\left(a_{0}\right)$ & 2.282 & 2.31 & & $\begin{array}{c}2.4 \\
(2.3)\end{array}$ & 2.32 \\
\hline$D_{e}^{+}(\mathrm{eV})$ & 6.78 & 5.51 & $\begin{array}{c}5.671 \\
(6.009)\end{array}$ & $\begin{array}{c}5.22 \\
(6.39)\end{array}$ & 6.27 \\
\hline$r_{t}^{+}\left(a_{0}\right)$ & 2.11 & 2.12 & & $\begin{array}{c}2.4 \\
(2.2)\end{array}$ & 2.22 \\
\hline $\operatorname{IP}\left(\mathrm{O}_{2}\right)_{r_{e}}(\mathrm{eV})$ & 12.3 & $\begin{array}{c}12.00 \\
(12.73)\end{array}$ & & $\begin{array}{l}8.26 \\
8.02\end{array}$ & 9.33 \\
\hline $\begin{array}{l}\operatorname{IP}(\mathrm{O})(\mathrm{eV}) \\
\left.E\left(\mathrm{O}^{3} P\right) \text { (a.u. }\right)\end{array}$ & $\begin{array}{r}13.6 \\
\ldots\end{array}$ & $\begin{array}{c}14.06 \\
-74.804\end{array}$ & $\begin{array}{c}-74.9174 \\
(-74.9193)\end{array}$ & $\begin{array}{c}9.35 \\
-74.509\end{array}$ & $\begin{array}{c}10.62 \\
-74.436\end{array}$ \\
\hline
\end{tabular}

"From Ref. 21.

bCF-CI calculation described in Sec. II B using the $[11,6 / 5,4]$ basis of Ref. 13 plus a $d$ polarization orbital. ( $\downarrow)$ In brackets, results obtained by using a different contraction scheme: $[11,6 / 5,3]$ and adding one diffuse $s\left(\zeta_{s}=0.092\right)$ and one diffuse $p$ orbital $\left(\zeta_{p}=0.059\right)$.

'MRD.CI data from Ref. 10. In brackets, Davidson's full-CI extrapolation (from Ref. 10).

${ }^{d} \mathrm{SCF}$-CI calculation described in Sec. II B using the STO-6G expansion of the single- $\zeta$ basis of Ref. 15 . In brackets, results obtained by adding a $d$ polarization orbital $\left(\zeta_{d}=0.8\right)$.

SCF-CI calculation described in Sec. II B using the scaled STO-6G basis of col. 4 (see Sec. IV A 1); the scaling factors are: $1.1,0.8$, and 1.0 for the $1 s, 2 s$, and $2 p$, respectively.

(ii) for the CI step: 300 CSF's describing all arrangements of eight (respectively, seven) electrons in the $3 \sigma_{g, u} 4 \sigma_{g, u}, 1 \pi_{g, u}^{x, y}$ MO's as well as the arrangements of two (respectively, one) electrons in the $1 \pi_{g}^{x, y}$ and $2 \pi_{g, u}^{x, y}$ MO's.

Comparison with the experimental data [Figs. 1(a) and 1 (b) and Table I, col.1) is quite disappointing. More extensive calculations, quoted in Ref. 10, using a few thousands CSF selected out of a set of about $10^{5}$ yield the result shown in Table I, col. 3. The well depth obtained from the latter calculations is underestimated by $0.7 \mathrm{eV}$ (respectively, $1.1 \mathrm{eV}$ ). To go beyond such results would require such large $\mathrm{CI}$ calculations for the $\mathrm{O}_{2} / \mathrm{O}_{2}^{+}$diatomics that the further treatment of any $\left(\mathrm{XO}_{2}\right)^{+}$collisional system would become hardly feasible.

Though minimal basis set expansions are generally frowned upon, we have considered whether, if amended in ad hoc manner they could be of some usefulness. The reason for this emanated from early calculations by Schaef$\mathrm{fer}^{14}$ and Beebe et al. ${ }^{15}$ who showed that full CI calculations with minimal Slater-type orbital bases could actually provide reasonable descriptions of the $\mathrm{O}_{2} / \mathrm{O}_{2}^{+}$potential energy curves. The number of CSF's implied in those calculations was only in the range of $10^{2}$. Table $I$, col. 4 and Figs. 1(a) and 1(b) show full CI results obtained with an STO- $6 \mathrm{G}^{16}$ expansion of the original $\mathrm{O}_{2}$ basis of Beebe et al. ${ }^{15}$ Adding a $d$-GTO $\left(\zeta_{d}=0.8\right)$ to this basis (which is also frowned upon $)^{17}$ and considering the above-described $\mathrm{CI}$ yielded $\mathrm{O}_{2} / \mathrm{O}_{2}^{+}$dissociation energies within $0.3 \mathrm{eV}$ of the experimental values [Table I, col. 5 and Figs. 1(a) and 1(b)]. Minimal basis sets have, however, two drawbacks. The first one is readily seen from Table I: the ionization potentials (IP) of both $\mathrm{O}_{2}\left(X^{3} \Sigma_{g}^{-}\right)$and $\mathrm{O}\left({ }^{3} P\right)$ are strongly underestimated. The second nastier feature for the planned $\left(\mathrm{XO}_{2}\right)^{+}$calculation is, of course, the well known basis set superposition error (BSSE): ${ }^{18}$ the $\mathrm{O}_{2} / \mathrm{O}_{2}^{+}$groups are better described with the triatomic basis than with their constrained diatomic minimal basis.

Hence, to summarize, the advantage of a minimal basis set, if one disregards O-O BSSE, is the computational ease it offers: both the number of two-electron integrals and the number of CSF's to be handled in a full, or nearly full, CI are well bounded. The main disadvantages are the uncontrolled effect of BSSE and the misplacement of $\mathrm{X}^{+}-\mathrm{O}_{2}$ levels with respect to $\mathrm{X}-\mathrm{O}_{2}^{+}$ones. On the other hand, with extended basis sets the mentioned BSSE is drastically reduced, but the size of the CI that is required to achieve a correct description of $\mathrm{O}_{2}^{(*)}$ and $\mathrm{O}_{2}^{+(*)}$ energy curves is excessive.

In the following we propose two procedures to correct the above-mentioned drawbacks. One handles the BSSE problem, while the other corrects misplacements of the separated-partner energy levels.

\section{DIAGONAL CORRECTIONS OF THE PVB-Cl ENERGY MATRIX}

\section{A. Correction of the atom-molecule BSSE}

Here we deal exclusively with basis set superposition errors arising from the approach of the atomic and molecular partners and disregard those effects arising from the approach of the atoms constituting the molecular partner itself.

The procedure consists in a counterpoise method $^{18}$ for a PVB-CI computational scheme. Its use is illustrated for the case of a state, labeled $\alpha$, of the $\mathrm{O}_{2}$ molecule. Let, for example, the relevant orbitals be expanded in the crude single- $\zeta+$ polarization basis of Sec. II and let us call $E_{\alpha}(r)$ 
the energy of the considered state obtained by the CI described there for the $\mathrm{O}-\mathrm{O}$ bond distance $r$.

We next define $\mathscr{E}_{\alpha}(R, r, \gamma)$ the energy of the considered state $\alpha$ when, in addition to the above-mentioned CI, one includes excitations to an atomic "ghost" placed at the point $R, \gamma$ from the molecule. ( $R$ is the center of mass to center of mass $\mathrm{H}-\mathrm{O}_{2}$ relative distance and $\gamma$ the $(r, R)$ angle). The ghost atom is obtained by annihilating the charge of the actual atomic partner and suppressing all of its electrons. We have of course, $\mathscr{E}_{\alpha}(R \rightarrow \infty, r, \gamma) \rightarrow E_{\alpha}(r)$. The energy change of the considered state caused by the enlargement of the basis for the geometry $R, r, \gamma$ is the BSSE,

$$
\Delta E_{\alpha}^{\mathrm{BSSE}}(R, r, \gamma)=\mathscr{E}_{\alpha}(R, r, \gamma)-E_{\alpha}(r) .
$$

The procedure is, of course, readily transposed to determine the BSSE for states of the atomic partner.

Ideally one wishes the mentioned additional excitations to be identical to those used in the actual PVB-CI calculation on the real triatomic system. Thus in calculating $\mathscr{E}_{\alpha}(R, r, \gamma)$, one should discard excitations to orbitals belonging to the occupied space of the real atomic partner. This is most easily achieved in a VB scheme since orbitals in VB-CSF's are specifically attributed to the molecular or the atomic partner. Another practical consideration has to do with the constraint imposed by the spatial and spin symmetries of the considered state $\alpha$. Obviously, because the atomic ghost carries no electron, only a part of the actual excitations in the real triatomic is compatible with that symmetry. Finally, on account that BSSE arises from the allowance offered to a given orbital of one partner to have components in a space complementary to that spanned by its original expansion basis, we have found it preferable to orthogonalize the orbitals of the ghost to those of the real partner in the process of determining the BSSE.

The thus determined BSSE for a given state $\alpha$ of the molecular (or atomic) partner is then used to modify the PVB-CI energy matrix: The diagonal elements corresponding to the dominant PVB-CSF's which make up the PVBCI state $\alpha$ are shifted by the amount $-\Delta E_{\alpha}^{\mathrm{BSSE}}(R, r, \gamma)$. Since the diagonalization of the modified PVB Hamiltonian matrix can be carried through after having affected the CI in each block $\alpha$, it is easy to convince oneself that this procedure is likely to remove most, if not all, of the BSSE in the states of the triatomic system correlating with state $\alpha$.

\section{B. Correction of misplaced energy levels of the separated partners}

As already pointed out in Secs. I and II owing to limited sizes of orbital and/or CSF expansion bases equilibrium properties, excitation energies, and ionization potentials of the separated partners may be more or less inaccurate. Such inaccuracies are detrimental to the description of ion-molecule charge transfer systems (Sec. I). Our contention is that, given a reasonable basis set size, instead of attempting to improve the above-mentioned properties by basis set enlargements, which may become prohibitive, one may try and take advantage of available accurate data from experiment or from previous large scale $a b$ initio calculations. The idea is to replace the actual Hamiltonian by another ad hoc one which when it acts on the approximate wave functions yields exact or nearly exact energies.

Consider the ground state and a few excited states of $\mathrm{O}_{2}$ and $\mathrm{O}_{2}^{+}$at a given bond distance $r$ (see Sec. IV B for details). We select a small number of the dominant configurations for these states in the largest $\mathrm{CI}$ we have decided to limit ourselves to. Let $\alpha$ be one of the considered states and $\left\{i_{\alpha}\right\}$ the corresponding set of dominant components. We introduce diagonal corrections $\Delta i_{\alpha}$ to the original Hamiltonian matrix and adjust these corrections by trial and error in such a way that the placement of the considered eigenstates $\alpha$ for the modified Hamiltonian comes the closest possible to that of the corresponding exact eigenstates. Similar shift procedures have been proposed in earlier contributions. ${ }^{19}$ The shift procedure is applied to each $\mathrm{O}-\mathrm{O}$ bond distance. If needed it is readily transposed to the adjustment of calculated levels of the atomic partner. The thus determined corrections are then used along with eventual $\Delta E^{\mathrm{BSSE}}(R, r, \gamma)$ corrections for every $R, r, \gamma$ geometry of the triatomic system.

\section{APPLICATION OF THE ${ }^{3} \mathbf{A}^{\prime \prime}$ STATES OF $\left(\mathrm{H}-\mathrm{O}_{2}\right)^{+}$}

\section{A. Technicalities}

\section{Basis sets}

Two calculations have been performed. The first (to be referred to as $B_{\min }$ ) made use of a single- $\zeta+$ polarization expansion basis set for the $\mathrm{O}$ atoms of $\mathrm{O}_{2}$. It was obtained as an STO-6G expansion of the STO basis of Beebe et al. ${ }^{15}$ augmented by a $d$ orbital $\left(\zeta_{d}=0.8\right)$. Owing to the results discussed in Sec. II this basis was modified by scaling ${ }^{16}$ in order to obtain an optimum compromise for the ionization potential and the equilibrium properties of $\mathrm{O}_{2}$ and $\mathrm{O}_{2}^{+}$.

The second calculation (to be referred to as $B_{\text {ext }}$ ) made use of the extended $(11,6 / 5,4)$ expansion basis of Ref. 13 for $O$ augmented by the same $d$ polarization orbital as above. Both calculations made use of the same $\mathrm{H}$ basis: a (4/1) $s$-CGTO (Ref. 20) augmented with a $p$ polarization orbital $\left(\zeta_{p}=0.5\right)$.

\section{The PVB-Cl calculations}

The SCF-MO of $\mathrm{O}_{2}\left(X^{3} \Sigma_{g}^{-}\right)$and $\mathrm{H}\left(1^{2} S\right)$ plus the virtuals ( 13 for $\mathrm{O}$ and 3 for $\mathrm{H}$ ) are orthogonalized in the order specified in Eq. (1) before generating ${ }^{3} A$ " PVBCSF's. The CI included the following excitation schemes: EI $-\mathrm{H}^{+}+\mathrm{O}_{2}$ : (a) All excitations relevant to a full valence $\mathrm{CI}$ for $\mathrm{O}_{2}$; the $3 \sigma_{u}$ orbital was considered to belong to the $\mathrm{O}_{2}$ valence shell in the construction of this block.

(b) All single excitations from occupied valence orbitals in the $\mathrm{O}_{2}$ ground state configuration to virtual orbitals of $\mathrm{O}_{2}$. 
EII $-\mathrm{H}+\mathrm{O}_{2}^{+}$: (a) All excitations relevant to a full valence $\mathrm{CI}$ for $\mathrm{O}_{2}^{+}$; as in $\mathrm{EI}(\mathrm{a})$, the $3 \sigma_{u}$ orbital is considered to belong to the $\mathrm{O}_{2}^{+}$valence shell in the construction of this block.

(b) All single excitations from occupied valence orbitals in the $\mathrm{O}_{2}^{+}$ground state configuration to virtual $\mathrm{O}_{2}$ orbitals, plus all single excitations from $1 s_{\mathbf{H}}$ to $\mathrm{H}$ virtuals.

This amounts to a total of 389 PVB-CSF's (128 for CSF's of type EI and 261 for CSF's of type EII).

\section{The $\mathrm{H}-\mathrm{O}_{2}$ BSSE}

The $\mathrm{H}-\mathrm{O}_{2}$ BSSE was only determined for calculations involving the basis set $B_{\min }$.

According to the above-described PVB-CI scheme, since there are no excitations from $\mathrm{O}_{2}^{+}$orbitals to $\mathrm{H}$ orbitals, BSSE can solely arise from excitations in group EII(b). It is thus obtained from a PVB-CI calculation, with a ghost $\mathrm{H}$ atom. The calculation proceeds exactly as for the actual triatomic system except for the set of oneelectron attraction integrals which is different. $\Delta E^{\mathrm{BSSE}}$ is determined from Eq. (2) for the lowest two eigenvalues at each of the considered $R, r, \gamma$ geometries. The $\Delta E_{X^{3} \Sigma_{g}^{-}}^{B S S E}(R, r, \gamma)$ obtained for the $\mathrm{O}_{2}$ ground state is then used to correct the diagonal element of the actual triatomic Hamiltonian matrix for the

$$
a_{1 \sigma_{g}}^{\prime 2} a_{1 \sigma_{u}}^{\prime 2} a_{2 \sigma_{g}}^{\prime 2} a_{2 \sigma_{u}}^{\prime 2} a_{3 \sigma_{g}}^{\prime 2} a_{1 \pi_{u}}^{\prime 2} a_{1 \pi_{u}}^{\prime 2} a_{1 \pi_{g}}^{\prime} a_{1 \pi_{g}}^{\prime \prime}
$$

PVB-CSF which by far dominates the isolated $\mathrm{O}_{2}$ ground state $\mathrm{CI}$ eigenvector. As an expediency, we used the second of the above-determined $\Delta E^{\mathrm{BSSE}}$ to correct in the actual triatomic calculation the 127 diagonal elements for the other CSF's in the groups $E I(a),(b)$.

\section{Adjustment of the $\mathrm{H}^{+}+\mathrm{O}_{2}^{(*)}$ and $\mathrm{H}+\mathrm{O}_{2}^{+(*)}$ asymptotes}

This adjustment follows the general lines described in Sec. III B. It is applied to both the $B_{\min }$ and $B_{\text {ext }}$ calculations. For the former case the corresponding level shifts add up to the BSSE corrections (Secs. III A, IV A 3).

Taking the $\mathrm{H}+\mathrm{O}_{2}^{+}\left(X^{2} \Pi_{g}\right)$ asymptotic level as the origin of energies we impose that the $\mathrm{H}^{+}+\mathrm{O}_{2}\left(X^{3} \Sigma_{g}^{-}\right)$ level and the lowest

$$
\begin{aligned}
& -\mathrm{H}+\mathrm{O}_{2}^{+*}\left({ }^{4} \Pi_{u}, \text { two }{ }^{2} \Pi_{u},{ }^{4} \Sigma_{g}^{-},{ }^{2} \Delta_{g},{ }^{2} \Sigma_{g}^{-},{ }^{2} \Phi_{u}\right) ; \\
& -\mathrm{H}^{+}+\mathrm{O}_{2}^{*}\left({ }^{3} \Delta_{u},{ }^{3} \Pi_{g, u},{ }^{3} \Sigma_{u}^{-},{ }^{3} \Delta_{g}\right)
\end{aligned}
$$

levels be correctly placed relative to this reference. As discussed in Refs. 3, 7, and 8 (see also Secs. I and IV B) these excited states are involved in the adiabatic distortion of levels (polarization and electron transfer) causing the well in the lowest ${ }^{3} A$ " state. Accurate experimental ${ }^{21}$ and/or $a b$ initio $^{22}$ data are available for those states.

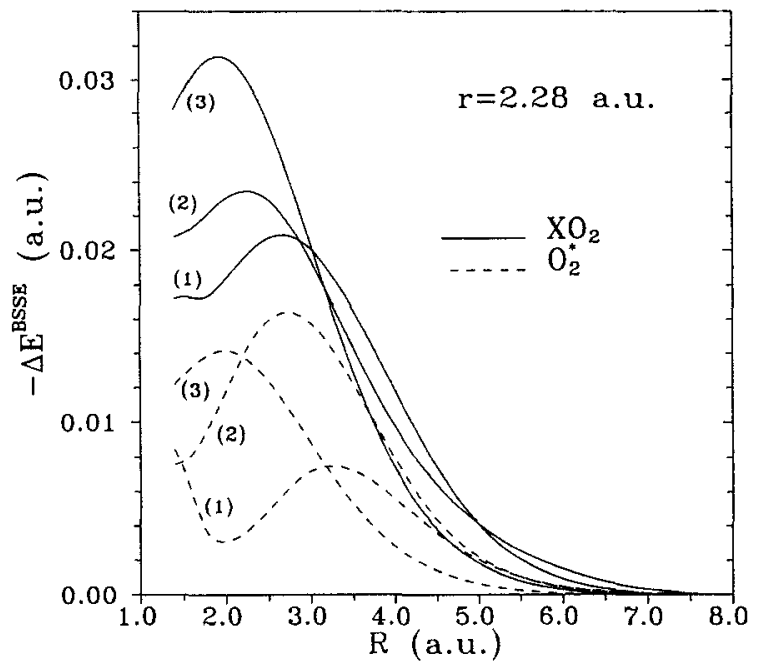

FIG. 2. Evolution of the BSSE correction $\Delta E^{\mathrm{BSSE}}(R, r, \gamma)$ in the $B_{\min }$ calculation as a function of the relative $\mathrm{H}-\mathrm{O}_{2}$ distance $R$ for the $\mathrm{O}-\mathrm{O}$ bond distance $r=2.28 a_{0}$ and three values of the relative angle $\gamma:(1) 0^{\circ}$, (2) $45^{\circ}$, and (3) $90^{\circ}$. Full lines: the $\Delta E_{X^{3} \Sigma_{g}^{-}}^{\mathrm{BSSE}}$ correction determined for the $\mathrm{O}_{2}$ ground state; dashed lines: $\Delta E^{\mathrm{BSSE}}$ used to correct the $\mathrm{H}^{+}+\mathrm{O}_{2}^{*}$ excited states of type EI(a),(b) in Sec. IV A 2.

\section{B. Results}

A hint at the importance and evolution of the BSSE correction in the $B_{\min }$ calculation may be got from Fig. 2 which shows selected cuts of the $\Delta E^{\mathrm{BSSE}}(R, r, \gamma)$ hypersurfaces. $\Delta E^{\mathrm{BSSE}}$ is seen to be reasonably small for all the investigated geometries.

Figure 3 shows the effects of the adjustment of the asymptotic energy levels discussed in Secs. III B and IV A 4. Figure 3(a) shows this effect for the $B_{\min }$ calculation after having corrected for the BSSE error. Figure 3(b) shows the corresponding result for the calculation $B_{\text {ext }}$. Figures 4(a)-4(c) illustrate samples of our final results incorporating all corrections for the lowest two ${ }^{3} A^{\prime \prime}$ states. It is quite remarkable that after so different corrections the $B_{\min }$ and $B_{\text {ext }}$ results agree so well. These figures also show the comparison of the $B_{\min }$ and $B_{\text {ext }}$ results with the EMP calculations of Ref. 8. The agreement for $r=2.28 a_{0}, \gamma=0$ and $45^{\circ}$ is most satisfactory. The discrepancy observed at $\gamma=90^{\circ}[\mathrm{Fig} .4(\mathrm{~b})]$ is presumably ascribable to a deficiency of the EMP calculation. Both Refs. 7 and 8 acknowledged the underestimation in $C_{2 v}$ symmetry of the well in the $1^{3} B_{1}$ potential energy curve. The detailed inspection of the procedure of Ref. 8 and the elements provided by the present study lead us to incriminate the misplacement in the EMP calculation of valence excited states of $\mathrm{O}_{2} / \mathrm{O}_{2}^{+}$ arising from excitations out of the $1 \pi_{u} \mathrm{MO}$. This explanation sheds light on the reason why this drawback shows up especially near $\gamma=90^{\circ}$. On account of the success of the EMP data in describing the charge transfer dynamics of the $\mathrm{H}^{+}+\mathrm{O}_{2}$ system, ${ }^{7}$ the nice overall agreement between the EMP data and the present results provides a good indication on the potential ability of the proposed proce- 


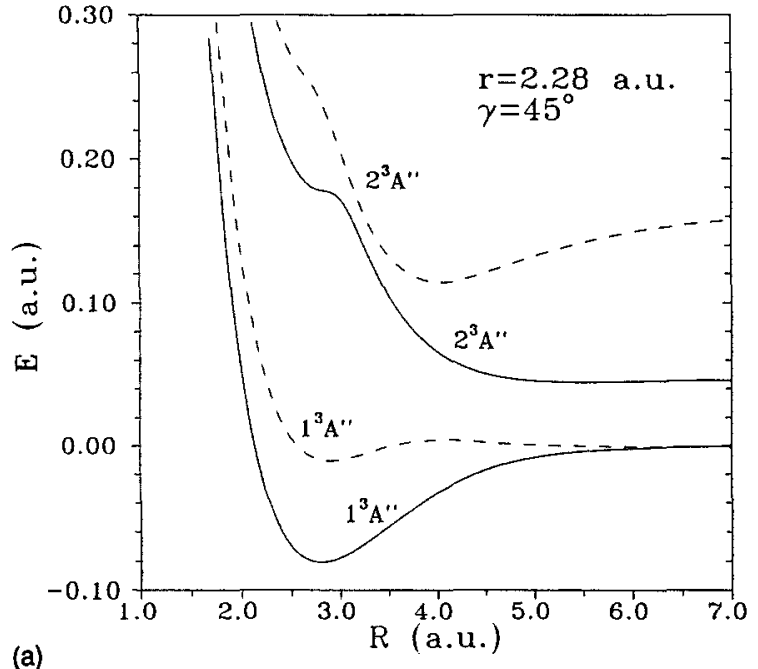

(a)

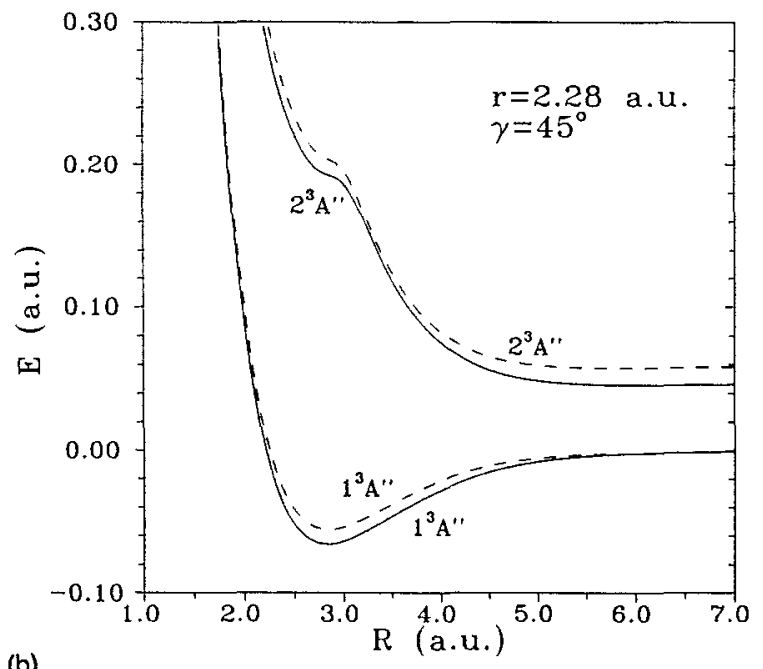

(b)

FIG. 3. Effects of the BSSE and $\triangle I P$ corrections on the lowest two ${ }^{3} A^{\prime \prime}$ states of $\left(\mathrm{HO}_{2}\right)^{+}$. The figure shows cuts of the two potential energy hypersurfaces for $r=2.28 a_{0}$ and $\gamma=45^{\circ}$. (a) $B_{\min }$ calculation. Dashed lines: after BSSE correction; full lines: after both BSSE and $\triangle$ IP corrections. (b) $B_{\text {ext }}$ calculation. Dashed lines: without $\Delta I P$ correction; full lines: after $\Delta \mathbf{I P}$ correction.

dures to produce appropriate data for the description of ion-molecule charge transfer.

Figure 5(a) shows a comparison of results obtained using the $B_{\text {ext }}$ basis for $r=2.53 a_{0}, \theta(\mathrm{HO}, \mathrm{OO})=106^{\circ}$ with data obtained from large scale $a b$ initio (MRD-CI) calculations. ${ }^{10} \mathrm{~A}$ first word of caution when inspecting this figure is that the MRD-CI results of Ref. 10 provide no information on the repulsive branch of the $2^{3} A$ " state connecting it at large $d_{\mathrm{OH}}$ distance with the $\mathrm{H}^{+}+\mathrm{O}_{2}\left(X^{3} \Sigma_{g}^{-}\right)$asymptote. This feature makes it difficult to understand why the $1{ }^{3} A^{\prime \prime}$ MRD-Cl curve rises more steeply than the presently calculated one at large $d_{\mathrm{OH}}$ distance. The shape of the MRD-CI $2{ }^{3} A$ " curve at large $d_{\mathrm{OH}}$ would have indicated whether the pinching of curves at the $1^{3} A^{\prime \prime}-2{ }^{3} A^{\prime \prime}$ avoided crossing ${ }^{8}$ is much narrower than the presently calculated one. The present ${ }^{3} A$ " well depth is found to be within $13 \%$ of MRD-CI one. Yet,
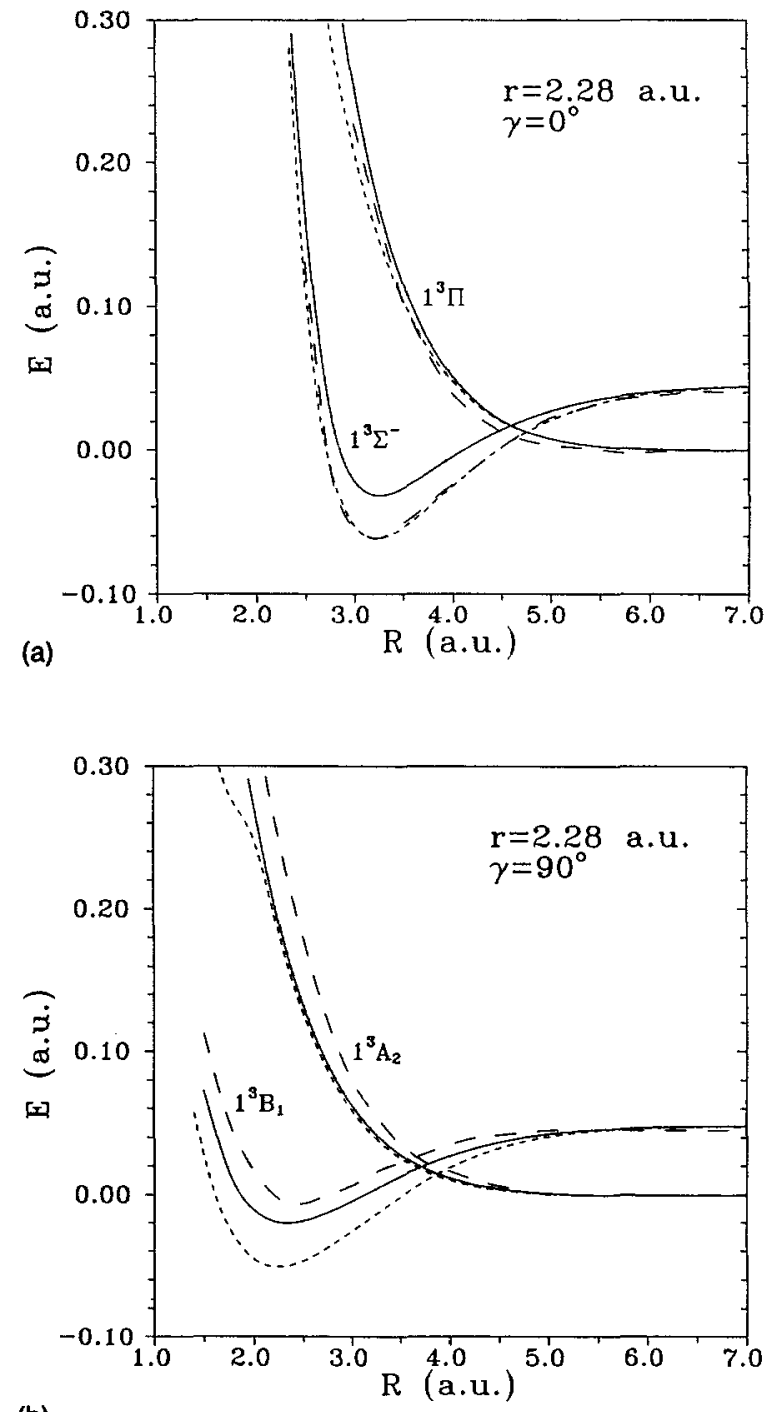

(b)

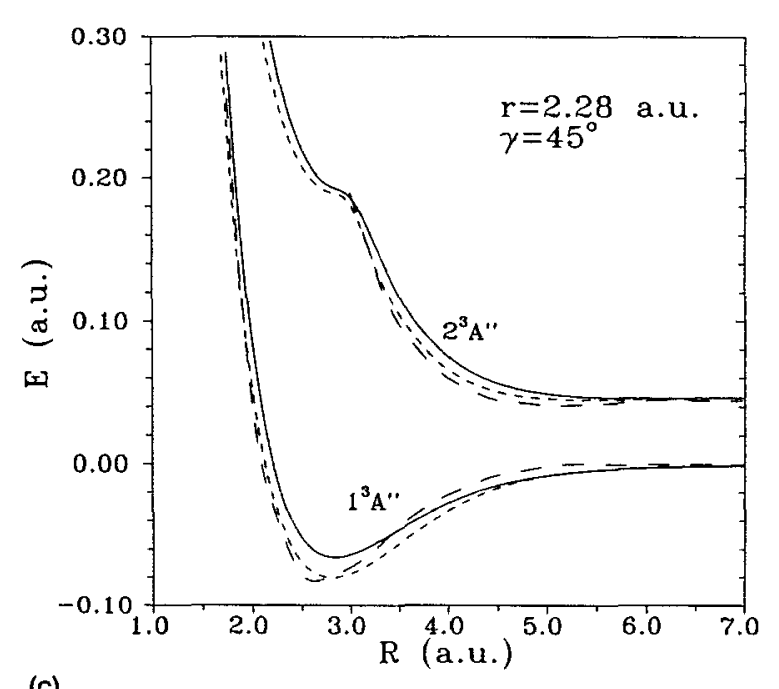

(c)

FIG. 4. Comparison of the present results for the lowest two ${ }^{3} A^{\prime \prime}$ states of $\left(\mathrm{HO}_{2}\right)^{+}$with EMP results of Ref. 8. Cuts of the two potential energy hypersurfaces for $r=2.28 a_{0}$ are shown for three values of the relative angle $\gamma$ : (a) $0^{\circ}$, (b) $90^{\circ}$, and (c) $45^{\circ}$. Full lines: $B_{\text {ext }}$ calculation, short dashed lines: $\boldsymbol{B}_{\min }$ calculation, long dashed lines: EMP data. 


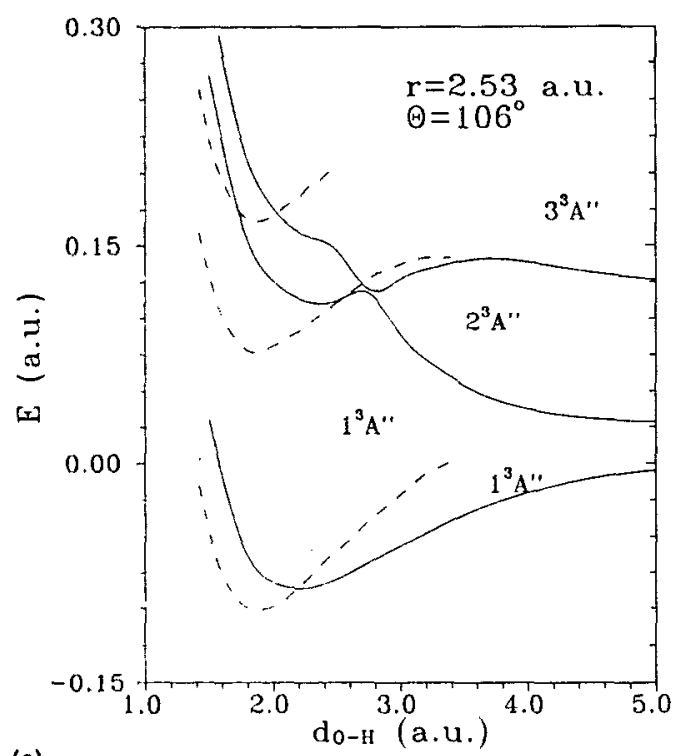

(a)

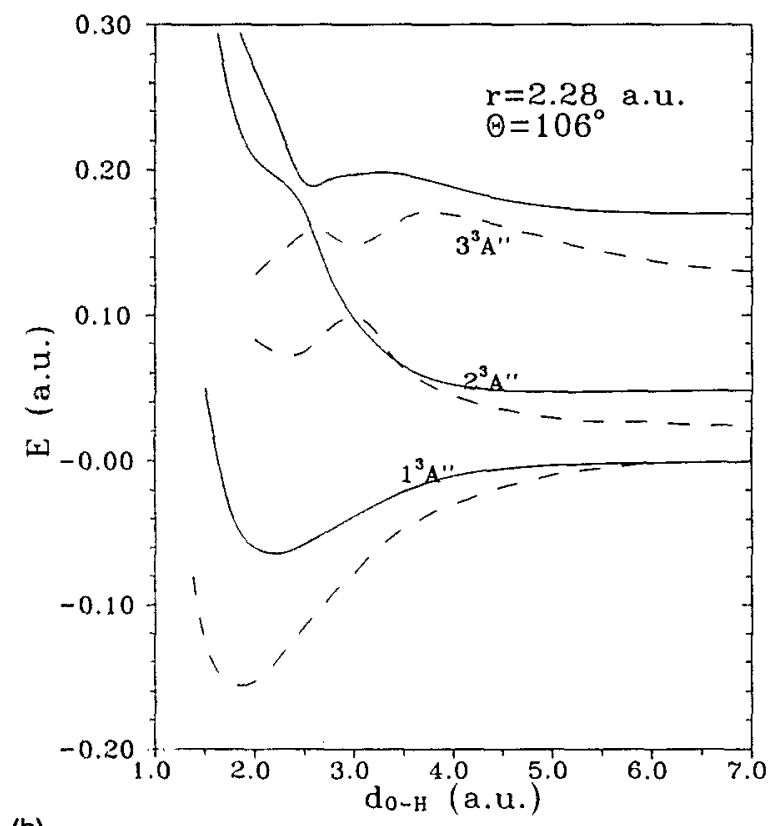

(b)

FIG. 5. Comparison of the present results for the lowest three ${ }^{3} A$ " states of $\left(\mathrm{HO}_{2}\right)^{+}$with MRD-CI results. (a) Cuts of the potential energy hypersurfaces for $r=2.53 a_{0}$ and $\theta=106^{\circ}$; full lines: present $B_{\text {ext }}$ results, dashed lines: results of Ref. 10. (b) Cuts of the potential energy hypersurfaces for $r \approx r_{e}\left(\mathrm{O}_{2}\right)$ and $\theta=106^{\circ}$; full lines: present $B_{\text {ext }}$ results for $r=2.28 a_{0}$ dashed lines: results of Schneider and Gdanitz for $r=2.29 a_{0}$ as reported in Ref. 6 . In these figures the abcissa $d$ is the distance between the $\mathrm{H}$ nucleus and the nearest $\mathrm{O}$ center.

there are not enough MRD-CI data points from the work of Vazquez et al. ${ }^{10}$ to decide whether there is a basic defect in the results of the present procedure or simply a displacement of properties to different $r, d_{\mathrm{OH}}, \theta$ geometries. On the whole, however, the correspondence of the two sets or results is acceptable when keeping in mind the important difference in computation labor involved in them.

Another set of MRCI results from unpublished calculations by Schneider and Gdanitz has been presented in Ref. 6. The reported data concern cuts through ${ }^{3} A^{\prime \prime}$ (re- spectively ${ }^{3} A^{\prime}$ ) potential energy surfaces for $r=2.29 a_{0}$ (respectively $\left.2.53 a_{0}\right) \theta(\mathrm{HO}, \mathbf{O O})=106^{\circ}$. The size of the orbital basis set (triple- $\zeta+$ diffuse + polarization functions) together with the CI dimension $(\sim 180000)$ led Schneider et $a l^{6}{ }^{6}$ to consider this large scale $a b$ initio calculation as a reference for the $\left(\mathrm{H}-\mathrm{O}_{2}\right)^{+}$system. The corresponding data, reproduced from Fig. 4 of Ref. 6, are shown together with the present $B_{\text {ext }}$ results in Fig. 5(b). The MRCI results of Ref. 6 are, however, somewhat confusing for a few reasons: (i) the asymptotic energy splitting of the $1^{3} A^{\prime \prime}$ and $2^{3} A^{\prime \prime}$ states underestimates the $\mathrm{H}+\mathrm{O}_{2}^{+}\left(X^{2} \Pi_{g}\right)-\mathrm{H}^{+}+\mathrm{O}_{2}\left(X^{3} \Sigma_{g}^{-}\right)$resonance energy defect by $0.68 \mathrm{eV}$; (ii) the well depth in that $1{ }^{3} A^{\prime \prime}$ curve is $4.12 \mathrm{eV}$, while both the experimental ${ }^{23}$ and the MRD-CI calculation of Vazquez et al. ${ }^{10}$ agreed on a smaller value: $2.88 \pm 0.046$ and $2.84 \mathrm{eV}$, respectively; and (iii) the shape of the $1^{3} A^{\prime \prime}$ curve at small $d_{\mathrm{OH}}$ distance was extrapolated in an unknown fashion using data from Ref. 10. These peculiarities thwarted a fair comparison with our results.

Figures 6(a)-6(c) show a more extensive view of our $B_{\text {ext }}$ results including excited ${ }^{3} A$ " states. The collinear [Fig. 6(a)] and T-shape [Fig. 6(b)] geometries provide an indication on the origin of the avoided crossings seen in Figs. 4 (c) and 5. These results bear the same qualitative appearance as those obtained in the EMP calculations. ${ }^{8}$ Yet closer inspection reveals that all the states in the EMP calculation are not correctly placed at infinity: particularly states having strong components on CSF's involving excitations out of the $1 \pi_{u}$ MO. The importance of the proper placement of these states in order to correctly account for the $1{ }^{3} B_{1}$ potential well in $C_{2 v}$ geometry has already been stressed in Sec. IV B 2. Given the particular care we have put on the placement of such levels in the separated partner limit we are confident that the present calculations provide a better description of the excited states than that obtained in Ref. 8. As in Sec. IV B 2, there is not enough data from the MRD-CI work $^{10}$ to discuss in perspective the comparison of excited states curves appearing in Fig. 5(a).

\section{CONCLUSION}

Two approximate procedures for calculating electronic energies of charge transfer systems have been tried on a prototype case: $\left(\mathrm{HO}_{2}\right)^{+}$. Both approaches are based on a PVB-CI scheme restricted to full valence CI of both the neutral and ionic partners augmented by single (polarization + electron transfer) excitations. To avoid the extensive $\mathrm{CI}$ that is required to accurately reproduce the separated partner properties in the ground and excited states we have had recourse to diagonal corrections of the PVB-CI Hamiltonian matrix which insure the exact placement of important energy levels at asymptotic atommolecule separation. With a view to further lighten the computational work, one of the procedures makes use of a single- $\zeta+$ polarization orbital expansion basis and is corrected for the relative atom-molecule BSSE. The nice outcome of this work is that results of the two procedures are in mutual accord and agree with earlier EMP data ${ }^{8}$ which have successfully been used in the treatment of the $\mathrm{H}^{+}+\mathrm{O}_{2}$ charge transfer dynamics. ${ }^{7}$ The present results 


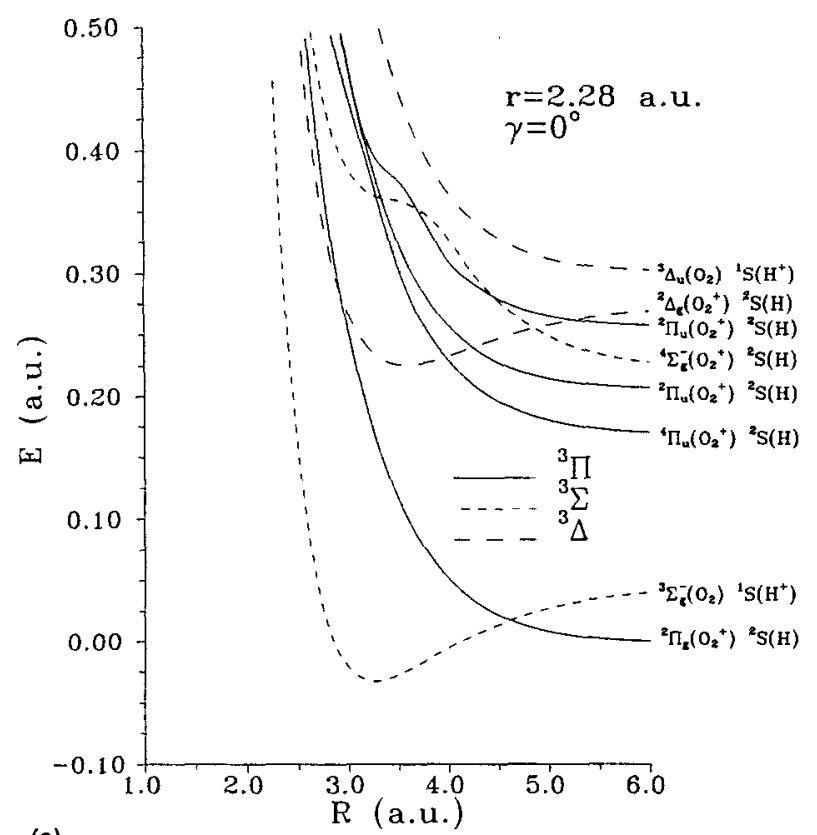

(a)

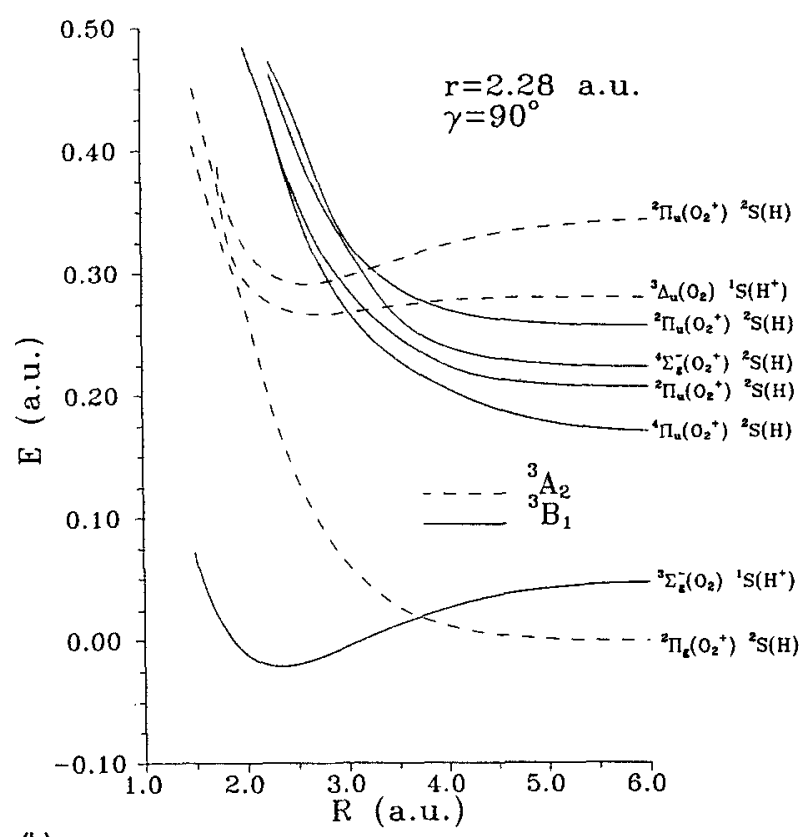

(b)

also acceptably reproduce qualitative features of fragmentary MRD-CI calculations of Vazquez et al. ${ }^{10}$ The proposed procedures are thus found to achieve a reasonable compromise between computational ease and accuracy. They provide, at the same time, information of comparable quality on several low lying excited states correlating with valence excited states of the separated partners. All of these characteristics make us think that these procedures will be most helpful when dealing with more complicated ionmolecule systems. We are inclined to think that of the two procedures tried, the one employing the extended basis is more appealing because it requires less manipulation. Yet the minimal basis approach is likely to be of some usefulness in the treatment of bulky ion-molecule systems.

Work is underway to apply these procedures to (rare gas $\left.-\mathrm{O}_{2}\right)^{+}$collision systems.

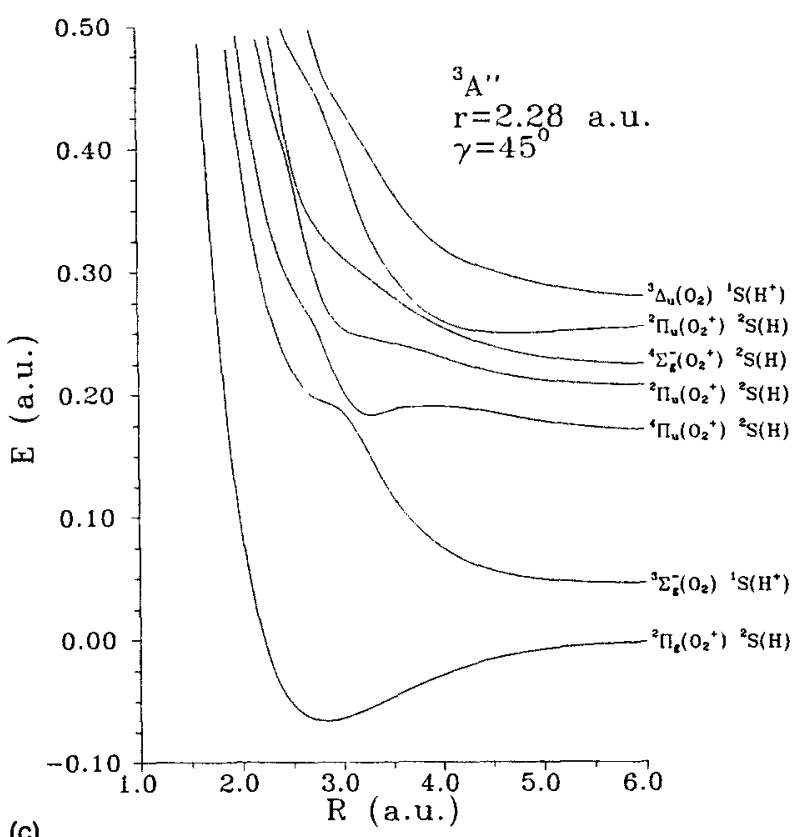

(c)

FIG. 6. Cuts of the present $B_{\mathrm{ext}}$ results for some of the lowest ${ }^{3} A^{\prime \prime}$ states of $\left(\mathrm{HO}_{2}\right)^{+}$for $r=2.28 a_{0}$ and (a) $\gamma=0^{\circ}$, (b) $\gamma=90^{\circ}$, (c) $\gamma=45^{\circ}$.

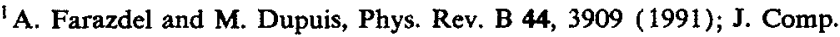
Chem. 12, 276 (1991).

${ }^{2}$ State-Selected and State-to-State Ion-Molecule Reaction Dynamics, edited by C. Y. Ng and M. Baer, Adv. Chem. Phys. Series Vol. LXXXII (Wiley, New York, 1992).

${ }^{3} \mathrm{~V}$. Sidis, in Ref. 2.

${ }^{4}$ Th. Glenewinkel-Meyer, Ch. Ottinger, P. Rosmus, and H.-J. Werner, Chem. Phys. 152, 409 (1991).

${ }^{5}$ (a) S. Scherbarth and D. Gerlich, J. Chem. Phys. 90, 1610 (1989); (b) O. Dutuit, Proceedings of the NATO-ASI on Fundamentals of Gas Phase Ion Chemistry, edited by K. R. Jennings (Kluwer Academic, Dordrecht, 1991); (c) E. E. Ferguson, M. Kriegl, R. Richter, P. Tosi, W. Federer, and W. Lindinger, Chem. Phys. Lett. 124, 583 (1986); (d) P. Tosi, M. Ronchetti, and A. Lagana, Chem. Phys. Lett. 136, 5 (1987); (e) M. F. Jarrold, L. Misev, and M. T. Bowers, J. Chem. Phys. 81, 4369 (1984).

${ }^{6}$ F. Schneider, L. Zülicke, F. Di Giacomo, F. A. Gianturco, I. Paidarova, and R. Polak, Chem. Phys. 128, 311 (1988). 
${ }^{7}$ M. Sizun, D. Grimbert, V. Sidis, and M. Baer, J. Chem. Phys. 96, 307 (1992).

${ }^{8}$ D. Grimbert, B. Lassier-Govers, and V. Sidis, Chem. Phys. 124, 187 (1988).

${ }^{9}$ M. Noll and J. P. Toennies, J. Chem. Phys. 85, 3313 (1986).

${ }^{10}$ G. J. Vazquez, R. J. Buenker, and S. D. Peyerimhoff, Mol. Phys. 59, 291 (1986).

"G. P. Raine, H. F. Schaeffer III, and N. C. Handy, J. Chem. Phys. 80, 319 (1984).

${ }^{12}$ C. Kubach and V. Sidis, Phys. Rev. A 14, 152 (1976).

${ }^{13}$ J. H. Dunning, J. Chem. Phys. 55, 716 (1971).

${ }^{14}$ H. F. Schaeffer III and F. E. Harris, J. Chem. Phys. 48, 1946 (1968).

${ }^{15}$ N. H. F. Beebe, E. W. Thulstrup, and A. Anderson, J. Chem. Phys. 64, 2080 (1976).

${ }^{16}$ R. F. Steward, J. Chem. Phys. 52, 431 (1970).
${ }^{17}$ H. F. Schaeffer III, The Electronic Structure of Atoms and Molecules: A Survey of Rigorous Quantum Mechanical Results (Addison Wesley, Reading, 1972).

${ }^{18}$ See, for instance, (a) S. F. Boys and F. Bernardi, Mol. Phys. 19, 553 (1970); (b) J. Van Lenthe, J. G. C. M. van Duijneveldt-van de Rijdt, and F. B. van Duijneveldt, Adv. Chem. Phys. 69, 521 (1987).

${ }^{19}$ G. Chambaud and B. Levy, J. Phys. B 22, 3155 (1989).

${ }^{20}$ S. Huzinaga, J. Chem. Phys. 42, 1293 (1965).

${ }^{21}$ (a) F. R. Gilmore, J. Quantum Spectrosc. Radiat. Trans. 5, 369 (1965); (b) P. Krupenie, J. Phys. Chem. Ref. Data 1, 423 (1972).

22 (a) R. P. Saxon and B. Liu, J. Chem. Phys. 67, 5432 (1977); (b) R. Klotz and S. D. Peyerimhoff, Mol. Phys. 57, 573 (1986).

${ }^{23}$ J. M. Dyke, N. B. H. Jonathan, A. Morris, and J. M. Winter, Mol. Phys. 44, 1059 (1981). 